\title{
Channel Sensing-Order Setting in Cognitive Radio Networks: A Two-User Case
}

\author{
Rongfei Fan and Hai Jiang, Member, IEEE
}

\begin{abstract}
This paper investigates the sensing-order problem in two-user multichannel cognitive medium access control. When adaptive modulation is not adopted, although brute-force search can be used to find the optimal sensing-order setting of the two users, it has huge computational complexity. Accordingly, we propose two suboptimal algorithms, namely, the greedy search algorithm and the incremental algorithm, which have comparable performance with that of brute-force search and have much less computational complexity. It is shown that, with a high probability, either suboptimal algorithm can reach an optimal point if a backoff mechanism is used for contention resolution. When adaptive modulation is adopted, it is observed that the traditional stopping rule does not lead to an optimal point in the two-user case. Furthermore, we demonstrate that the adoption of adaptive modulation affects the optimal sensing-order setting of the two users, compared with the case without adaptive modulation. These findings imply that the stopping rule and the sensing-order setting should be jointly designed from a systematic point of view.
\end{abstract}

Index Terms-Cognitive radio, multiple access.

\section{INTRODUCTION}

$\mathbf{I}$ $\mathrm{N}$ DIFFERENT regions of the world, the majority of the appropriate wireless spectrum has been licensed to various applications. Potential spectrum shortage in the future will bring a big challenge in the development of new wireless networks. Recently, a spectrum-agile technology, namely, cognitive radio [1]-[4], has emerged to alleviate the spectrum scarcity problem, which is mainly motivated by the significant underutilization of the licensed spectrum at a time or at a location. In a cognitive radio network, secondary users (i.e., unlicensed users) can get access to the licensed spectrum when there is no transmission/reception activity of primary users (i.e., licensed users). This means that secondary users have a lower priority in the spectrum access, which will guarantee that primary users are not affected. Due to the lower spectrum access priority, secondary users should sense possible primary activities before their own transmissions [5]-[7].

As a number of potential frequency bands (referred to as channels in the sequel) may be available, a secondary user also has flexibility in selecting one from the multiple channels to transmit. This is referred to as multichannel cognitive medium access control, which has recently received much attention.

Manuscript received February 21, 2009; revised May 15, 2009. First published September 29, 2009; current version published November 11, 2009. This work was supported by the Alberta Ingenuity Fund, Alberta, Canada. The review of this paper was coordinated by Dr. Y.-C. Liang.

The authors are with the Department of Electrical and Computer Engineering, University of Alberta, Edmonton, AB T6G 2V4, Canada (e-mail: rongfei@ualberta.ca; hai.jiang@ece.ualberta.ca).

Digital Object Identifier 10.1109/TVT.2009.2027712
In [8], secondary users cooperate to sense the potential channels, share the sensing results, and negotiate their transmissions via a control channel. A partially observable Markov decision process is used in [9] to model the idle/busy states of each potential channel. Multiarmed bandit problems are used in [10] and [11] to model the dynamic channel sensing and selection. In the research efforts in [9] and [10], a secondary user first selects a channel to sense and then transmits if the channel is sensed to be idle or keeps silent for a period if the channel is sensed to be busy. On the other hand, a secondary user can sequentially and continuously sense selected channels until one channel is identified to be idle. In this context, the sensing order of the channels and the related stopping rule [12], [13] (i.e., when to stop sensing and start transmission) are essential. In [14], the optimal channel-sensing strategy including the sensing order and the stopping rule is derived for a single-user case, with an assumption that recall (i.e., the secondary user can go back to access a previously sensed channel) and guess (i.e., the secondary user is permitted to access a channel that has not yet been sensed) are allowed. In [15], it is shown that prohibitive complexity is needed to obtain the optimal channel-sensing strategy, and polynomial-complexity algorithms with parameter $\epsilon$ are given, whose rewards are at most $\epsilon$ less than that of the optimal strategy. Recall and guess are permitted. In [16], the optimal sensing-order problem is investigated for a single-user case where neither recall nor guess is allowed. It is shown that, in some special scenarios, a simple sensing order does exist. It can be seen that [14]-[16] all focus on the optimality of a single user inside a time frame, assuming that the state (e.g., free/busy, channel capacity, etc.) of a channel is independent of other channels' states, as well as independent of the states of the same channel in other time slots. In [17], a centralized coordinator is used to discover the spectrum opportunities for a cognitive radio network. The optimal channel sensing order is derived for channels with homogeneous capacities, and it is shown that the problem of the optimal sensing order for channels with heterogeneous capacities is NP-hard. In [18], all the channels have the same free probabilities. Therefore, the sensing-order problem is not applicable. The optimal stopping rule is derived.

From the aforementioned discussion, it can be seen that the optimal sensing-order problem and the related stopping rule problem are investigated in the literature, either for a single-user case or in a centralized system, where only one sensing order (for the single user or for the coordinator) is considered. The problem is still open for a distributed multiuser case, where the channel sensing and estimation are performed by the users in a distributed manner (i.e., each user has a unique sensing order). 
As a follow-up of the research in [16], as well as our first step to solving the sensing-order problem with multiple users, here, we target at a cognitive radio network with two secondary users. The contribution of this paper is twofold. First, for the case without adaptive modulation, although brute-force search can be used to find an optimal sensing-order setting of the two users, it has huge computational complexity. Accordingly, we propose two low-complexity suboptimal algorithms that have comparable performance with that of brute-force search. It is shown that the system throughput of either suboptimal algorithm is close to the maximal throughput obtained through brute-force search, and the relative difference of the system throughput (of either suboptimal algorithm) from the maximal throughput is bounded by $5 \%$ in numerical examples. In addition, with a high probability, either suboptimal algorithm can reach an optimal point if a backoff mechanism is used for contention resolution. Second, for the case with adaptive modulation, we demonstrate that the traditional stopping rule is not optimal in a two-user case, although it is optimal in a single-user case. We also demonstrate that the adoption of adaptive modulation affects the optimal sensing-order setting of the two users. Our results imply that, unlike a single-user case, the optimal sensing order and the stopping rule in a two-user case should be jointly designed from a systematical point of view.

The rest of this paper is organized as follows. Section II describes the system model. The following four sections are devoted to the case without adaptive modulation: Section III gives some examples, Sections IV and V propose two suboptimal algorithms, respectively, and Section VI presents numerical results. Section VII discusses the impact of adaptive modulation on the optimal system configuration, followed by concluding remarks in Section VIII.

\section{SySTEM MODEL}

Consider a cognitive radio network with a coordinator and two secondary users, ${ }^{1}$ i.e., users 1 and 2 . For the network, there are a number $N$ of potential channels, with channel indices $1,2, \ldots, N$, respectively. Each user can sense one channel at a time. Each user has a sensing order, i.e., $\left(a_{1}, a_{2}, \ldots, a_{N}\right)$ for user 1 and $\left(b_{1}, b_{2}, \ldots, b_{N}\right)$ for user 2 , which are permutations of $(1,2, \ldots, N)$. The sensing position of a channel with a user is defined as the position of the channel in the user's sensing order. Therefore, channel $a_{k}$ 's (or $b_{k}$ 's) sensing position with user 1 (or user 2) is $k$.

Similar to the research in [9], [10], [14], and [16], a slottedtime structure is adopted here. The network is assumed to be synchronous, and time is partitioned into slots, each with unit length. In each time slot, each channel is either occupied by primary activities for the whole slot duration or free of primary activity for the whole slot duration. For channel $i \in$ $\mathcal{N}=\{1,2, \ldots, N\}$ in a slot, it is free of primary activity with probability $\theta_{i} \in(0,1)$, which is referred to as the primary-free probability of channel $i$ in the sequel. For each channel, the primary activity state (idle or busy) in a slot is independent of the states in other slots and is independent of the primary

\footnotetext{
${ }^{1}$ By “secondary users," we mean secondary transmitters.
}

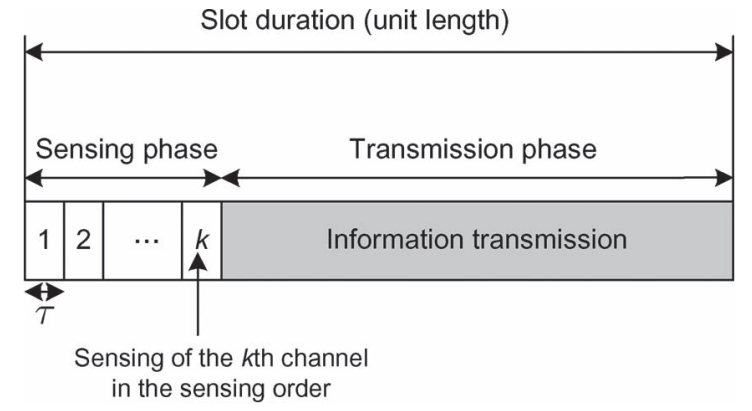

Fig. 1. Slot structure (when a secondary user stops after its $k$ th sensing).

activity state of any other channel in any slot as well. A similar assumption is also taken in [14]-[16]. For simplicity of presentation, we assume that $\theta_{i} \geq \theta_{i+1}, \forall i \in \mathcal{N} \backslash\{N\}$.

A coordinator exists in the cognitive radio network. The coordinator keeps scanning the $N$ channels at all times and estimates the primary-free probability of each channel. Based on the estimated primary-free probabilities, the coordinator determines the sensing orders of the two users and announces the sensing orders to the two users. Note that the primaryfree probabilities may be time varying. Therefore, new sensing orders will be determined and updated to the two users upon significant changes in the estimated primary-free probabilities. In this paper, we are interested in how the coordinator determines the sensing orders of the two users, with any specific estimated $\left\{\theta_{1}, \theta_{2}, \ldots, \theta_{N}\right\}$.

At the beginning of each slot, a user first sequentially senses the channels according to its sensing order, until it finds a free channel and transmits in the channel in the remaining time of the slot. If a user decides to access a channel (i.e., starts its transmission if a contention-resolution strategy is not adopted or starts its channel contention procedure if a contentionresolution strategy is adopted), we say that the user stops at that channel. This procedure is repeated in each of the following slots, i.e., each user repeats sensing the channel(s) and choosing a channel to transmit in each slot. No recall or guess is allowed. The slot structure is shown in Fig. 1, in which a user stops after its $k$ th sensing and starts transmission until the end of the slot. The fraction of time (in a slot) required for sensing a channel is denoted by $\tau$. Therefore, each slot consists of a sensing phase (with maximal duration $N \tau$ ) and a transmission phase (with minimal duration $1-N \tau$ ). If a user stops at the $k$ th channel in its sensing order, the lengths of the sensing phase and transmission phase are $k \tau$ and $1-k \tau$, respectively. The effectiveness of a slot is defined as the ratio of the transmission phase length to the slot length. Therefore, if a user stops at the $k$ th channel in its sensing order, the effectiveness is

$$
c_{k}=1-k \tau
$$

Energy detection and feature detection are two popular sensing schemes. The sensing duration for a channel in energy detection may be less than $1 \mathrm{~ms}$, while the sensing duration in feature detection may be more than $20 \mathrm{~ms}$ [19]. In this paper, each user may sequentially sense up to $N$ channels in a slot. Thus, a short sensing duration for each channel is required. 
Therefore, this research is valid only when energy detection is used in channel sensing.

If the two users simultaneously decide to stop at the same channel (i.e., the two users sense the same channel in their $k$ th sensing, find that the channel is primary-free, and decide to access the channel), one of the following three contentionresolution strategies will apply.

1) FAIL_THEN_CONTINUE: Each user uses a backoff mechanism to avoid possible collision (e.g., similar to the IEEE 802.11 and its variants [20]-[22], where each user can pick up a random backoff time before its channel access $^{2}$ ). One user wins in the contention and transmits in the channel until the end of the slot. The other user (which fails in the contention) continues to sense other channels in the slot according to its sensing order.

2) FAIL_THEN_QUIT: Similar to FAIL_THEN_CONTINUE, the two users use a backoff mechanism to avoid possible collision, and one user wins in the contention and transmits in the channel until the end of the slot. However, the other user (which fails in the contention) quits its subsequent sensings in the slot (i.e., loses its chance to transmit and earns no reward in the slot). Note that, compared with FAIL_THEN_CONTINUE, FAIL_THEN_QUIT is not practical. It is adopted here only as an extreme case.

3) COLLIDE: No backoff mechanism is applied. When the two users simultaneously decide to stop at the same channel, they both transmit in the channel until the end of the slot, and thus, a collision happens. In other words, no user can earn rewards in the slot due to the collision.

If one user decides to stop at a channel, it may select a transmission rate with or without adaptive modulation. Without adaptive modulation, the transmission rate of each user is fixed, which is denoted by $R$. With adaptive modulation, the transmitter can select a rate according to its SNR in the channel.

In the following, first, the case without adaptive modulation is discussed in Sections III-VI, and then, the case with adaptive modulation is investigated in Section VII.

\section{Some Examples Without Adaptive Modulation}

If adaptive modulation is not adopted, a user will decide to stop at a channel if the channel is sensed to be free. Here, "a channel is (sensed to be) free" means that the channel is primary-free and is not occupied by the other user in its prior sensings in the slot. We define the reward of a user as the information bits it can transmit at a slot. Therefore, if a user stops at its $k$ th channel in its sensing order, the reward is

$$
U_{k}=R \cdot(1-k \tau) .
$$

The system throughput is the summation of the two users' rewards, and our objective is to find an optimal sensing-order setting of the two users to achieve the maximal system throughput.

\footnotetext{
${ }^{2}$ Similar to the IEEE 802.11, a backoff slot duration can be $20 \mu \mathrm{s}$. Therefore, if the contention window size is bounded (say $\leq 5$ ), the backoff overhead is also bounded by a value that is much less than the sensing duration. This means that backoff does not cause significant overhead.
}

In this section, we use some examples to demonstrate the factors that affect the optimal sensing-order setting of the two users. In the examples, a cognitive radio network with $N=4$ potential channels is considered. The sensing time for each channel is $\tau=0.1$.

Example 1: The primary-free probabilities of the four channels are $\theta_{1}=0.9, \theta_{2}=0.8, \theta_{3}=0.7$, and $\theta_{4}=0.6$, respectively. FAIL_THEN_QUIT is the contention-resolution strategy.

By brute-force search, we can obtain the optimal sensingorder setting of the two users, which is $(1,4,3,2)$ for user 1 and $(2,3,4,1)$ for user 2 . This setting is reasonable because the four primary-free probabilities are comparable with each other. Therefore, if a user picks up a channel to sense, the other user will pick up another.

Example 2: The primary-free probabilities of the four channels are $\theta_{1}=0.9, \theta_{2}=0.8, \theta_{3}=0.7$, and $\theta_{4}=0.1$, respectively. FAIL_THEN_QUIT is the contention-resolution strategy.

By brute-force search, the optimal sensing-order setting is $(1,3,4,2)$ for user 1 and $(2,3,4,1)$ for user 2 , which is different from that in Example 1. This is because $\theta_{4}$ is much less than $\theta_{3}$. Consider the moment when user 2 is selecting ${ }^{3}$ its second channel to sense (among channels 1, 3, and 4, since channel 2 has been selected as the first channel in the sensing order). User 2 knows ${ }^{4}$ that the first two channels in user 1's sensing order are 1 and 3, respectively. From user 2's point of view at its second sensing, we observe the following.

1) Channel 1 is always busy, since channel 1 should be occupied by either primary users or user 1 . Therefore, if channel 1 is selected as user 2's second sensing, no reward is earned.

2) Consider the case in which user 2 selects channel 3 as its second sensing: When both channel 1 and channel 3 are primary-free (with probability $\theta_{1} \theta_{3}$ ), user 2's reward at its second sensing is $U_{2}$; when channel 1 is primarybusy and channel 3 is primary-free (with probability $\left.\left(1-\theta_{1}\right) \theta_{3}\right)$, user 2's reward at its second sensing is $0.5 U_{2}$ (where the term 0.5 means the probability that user 2 wins the contention with user 1 in channel 3); otherwise, the reward is zero. On the other hand, when channel 1 is primary-busy and channel 3 is primary-free, user 1 has a reward loss (compared with the case where user 2 does not select channel 3 in its second sensing), given by ( $1-$ $\left.\theta_{1}\right) \theta_{3} \cdot 0.5 \cdot U_{2}$. Therefore, if user 2 selects channel 3 in its second sensing, the additional reward to the system is given by $\theta_{1} \theta_{3} U_{2}+\left(1-\theta_{1}\right) \theta_{3} \cdot 0.5 U_{2}-\left(1-\theta_{1}\right) \theta_{3}$. $0.5 \cdot U_{2}=0.63 U_{2}$.

3) Channel 4 is free with probability $\theta_{4}=0.1$. If user 2 selects channel 4 in its second sensing, the additional reward to the system is $0.1 U_{2}$.

Therefore, user 2 selects the channel with the maximal additional reward to the system, i.e., channel 3. Both users select channel 3 as the second channel in their sensing orders. If the two users both proceed to sense channel 3, one will succeed

\footnotetext{
${ }^{3}$ For presentation simplicity, when we say "a user selects a channel," we mean that the coordinator selects the channel for the user.

${ }^{4}$ For presentation simplicity, here, "user 2 knows" actually means that "the coordinator knows."
} 
in the contention, and the other will quit subsequent sensings in the slot (i.e., lose the chance to sense other channels in the slot). However, the probability of this event is quite small, i.e., $\left(1-\theta_{1}\right)\left(1-\theta_{2}\right)=0.02$ in the example. Therefore, considering the probabilities and rewards/costs of all events, it is optimal that both users select channel 3 as their second channel to sense. In the next section, a detailed procedure is given regarding how to model the cost when the two users contend in the same channel and how to determine the sensing orders of the two users.

Example 3: The primary-free probabilities of the four channels are the same as those in Example 2, i.e., $\theta_{1}=0.9$, $\theta_{2}=0.8, \theta_{3}=0.7$, and $\theta_{4}=0.1$. However, COLLIDE is the contention-resolution strategy.

By brute-force search, the optimal sensing-order setting is $(1,4,3,2)$ for user 1 and $(2,3,4,1)$ for user 2 , which is different from that in Example 2. If the optimal sensingorder setting in Example 2 is applied, although the probability that both users simultaneously proceed to sense channel 3 is small, the cost of this event (i.e., no user will gain any reward in the slot) is significant. A mathematical model for the cost is given in next section. Therefore, the optimal sensing-order setting in Example 2 is no longer optimal when COLLIDE is the contention-resolution strategy.

From the examples, it can be seen that both the primaryfree probabilities of the channels and the contention-resolution strategy will affect the optimal sensing-order setting.

Generally, brute-force search can be used to find an optimal sensing-order setting of the two users but with significant complexity. If the complexity to calculate the average system throughput with a specific sensing-order setting is $O(1)$, then the complexity of brute-force search is $O\left((N !)^{2}\right)$. This paper targets at algorithms that have much less complexity and have comparable performance with that of brute-force search, as discussed in the following two sections.

\section{GREedy SEARCH Algorithm}

From Example 2 in the preceding section, we have the following observation. For a target user to determine which channel to sense in its $k$ th sensing, when a channel has already been in the other user's prior $(\leq k)$ sensing positions, the target user should estimate the probability that the channel is accessed by the other user. From Examples 2 and 3, it can be seen that the cost should be considered when the two users both proceed to sense the same channel at the same time. Based on these observations, we propose a suboptimal algorithm, namely, the greedy search algorithm, to select the sensing orders of the two users, i.e., $\mathbf{A}=\left(a_{1}, a_{2}, \ldots, a_{N}\right)$ for user 1 and $\mathbf{B}=$ $\left(b_{1}, b_{2}, \ldots, b_{N}\right)$ for user 2 .

The greedy search algorithm consists of $N$ rounds. In the $k$ th round, we determine $a_{k}$ and $b_{k}$. When user 1 (or 2) is selecting $a_{k}$ (or $b_{k}$ ) among channels not in its prior sensing positions, the user estimates the probability that each channel is free of primary activities and has not been accessed by the other user. The user also accordingly assigns each channel a reward. Then, the channel with the maximal reward is selected as $a_{k}$ (or $b_{k}$ ). The detailed selecting procedure is described as follows.
Let $\mathbf{A}$ and $\mathbf{B}$ denote the channel-selection vectors of users 1 and 2, respectively, which will be updated after each round. Let $\mathcal{A}$ and $\mathcal{B}$ denote the sets of channels in $\mathbf{A}$ and $\mathbf{B}$, respectively. Therefore, after the $k$ th round, $|\mathbf{A}|=|\mathbf{B}|=|\mathcal{A}|=|\mathcal{B}|=k$. Note that $\mathcal{A}$ and $\mathcal{B}$ have the same elements as $\mathbf{A}$ and $\mathbf{B}$, respectively. The difference is that $\mathcal{A}$ and $\mathcal{B}$ are sets, while $\mathbf{A}$ and $\mathbf{B}$ are vectors.

\section{A. Round-1 Procedure}

At the beginning, set $\mathcal{A}=\mathcal{B}=\emptyset$ (empty set). We first select $a_{1}$ for user 1 . We need to select a channel from $\overline{\mathcal{A}}=\mathcal{N} \backslash \mathcal{A}$. For each channel $i \in \overline{\mathcal{A}}$, we denote $G_{i}^{(1)}(1)$ as the reward user 1 can add to the system through sensing channel $i$ in its first sensing, which is given by $G_{i}^{(1)}(1)=\theta_{i} U_{1}$, where $U_{1}$ is given in (2). In the sequel, we use superscript $(\cdot)$ to represent the user index and the subscript to represent channel index (except for $c_{i}$ and $U_{i}$, where the subscript $i$ stands for the $i$ th sensing in a slot).

Then, the first channel to be sensed by user 1 is selected as

$$
a_{1}=\arg \max _{i \in \overline{\mathcal{A}}} G_{i}^{(1)}(1)
$$

and after that, we have $\mathbf{A}=\left(a_{1}\right)$ and $\mathcal{A}=\left\{a_{1}\right\}$.

Next, we select $b_{1}$ for user 2 . We need to select a channel from $\overline{\mathcal{B}}=\mathcal{N} \backslash \mathcal{B}$. If user 2 also selects $a_{1}$, it does not add any reward to the system (compared with the case when user 2 does not select $a_{1}$ ), because user 1 has already selected $a_{1}$. Therefore, we have

$$
b_{1}=\arg \max _{i \in \overline{\mathcal{B}} \backslash\left\{a_{1}\right\}} G_{i}^{(2)}(1)
$$

and after that, we have $\mathbf{B}=\left(b_{1}\right)$ and $\mathcal{B}=\left\{b_{1}\right\}$.

\section{B. Round- $k(\in\{2,3, \ldots, N-1\})$ Procedure}

At round $k \in\{2,3, \ldots, N-1\}$, we should determine which user we should start with. To achieve a certain level of fairness, ${ }^{5}$ we start with the user that has a less cumulative reward in the prior $k-1$ rounds, which is given by

$$
\theta_{a_{1}} U_{1}+\left(1-\theta_{a_{1}}\right) \theta_{a_{2}} U_{2}+\cdots+\left(\Pi_{i=1}^{k-2}\left(1-\theta_{a_{i}}\right)\right) \theta_{a_{k-1}} U_{k-1}
$$

for user 1 or

$$
\theta_{b_{1}} U_{1}+\left(1-\theta_{b_{1}}\right) \theta_{b_{2}} U_{2}+\cdots+\left(\Pi_{i=1}^{k-2}\left(1-\theta_{b_{i}}\right)\right) \theta_{b_{k-1}} U_{k-1}
$$

for user 2 .

Without loss of generality, assume that user 1 has a less cumulative reward, and thus, we should first select $a_{k}$ from $\overline{\mathcal{A}}$ and then select $b_{k}$ from $\overline{\mathcal{B}}$.

In the sequel, for each user, the primary-free probability of a channel means the probability that the channel is free of primary activity, and the free probability of a channel means

\footnotetext{
${ }^{5}$ It is hard to achieve strict fairness between the two users in a slot, since throughput maximization and strict fairness are two conflicting objectives in a short duration [23], [24]. However, The fairness of the two users can be guaranteed among a number of slots. This can be achieved, for example, through switching the sensing orders of the two users after a short period.
} 
the probability that the channel is free of either primary activity or the other secondary user's activity.

1) Selection of $a_{k}$ : To select $a_{k}$, we need to know the free probability (from user 1's viewpoint) of each channel $i \in \overline{\mathcal{A}}$. If $i \notin \mathcal{B}$, then channel $i$ 's free probability is the same as its primary-free probability, i.e., $\theta_{i}$. However, if $i \in \mathcal{B}$, channel $i$ is free for user 1 at its $k$ th sensing only when there is no primary activity in channel $i$ (with probability $\theta_{i}$ ) and user 2 does not proceed to sense channel $i$. To know the probability that user 2 does not proceed to sense channel $i$, we first update the primary-free probability of each channel in $\mathcal{B}$. Note that, for each channel $i, \theta_{i}$ is its initially known primary-free probability. When user 1 proceeds to sense $a_{k}$, we have more information that user 1 does not stop at channel $a_{1}, a_{2}, \ldots$, or $a_{k-1}$. The information can provide a more accurate estimation of the primary-free probabilities of the channels. We use the following example to explain.

As an example, assume that we have $N=6$ channels with the initially known primary-free probability for channel $i$ being $\theta_{i}$. Furthermore, we have determined that $\left(a_{1}, a_{2}, a_{3}\right)=$ $(1,3,4)$ and $\left(b_{1}, b_{2}, b_{3}\right)=(2,4,3)$. Now, we need to determine $a_{4}$. When user 1 proceeds to sense channel $a_{4}$, we know that it has already sensed channel 3 but has not stopped at channel 3 . Furthermore, if user 2 also senses channel 3, it should be later than user 1's sensing of channel 3, because channel 3 is second in user 1's sensing order, and third in user 2's sensing order. These two facts determine that channel 3 should be occupied by primary activities, i.e., $\theta_{3}^{*}=0$ in this example. We use superscript ${ }^{*}$ to denote the updated primary-free probability.

The detailed primary-free probability update procedure, as shown in the Appendix, is repeated from channel $b_{1}$ to $b_{k-1}$. In the Appendix, $g(i, \mathbf{B})$ is used to denote the position of $i$ in $\mathbf{B}$ if channel $i$ is in $\mathbf{B}$. In other words, channel $i$ is at the $g(i, \mathbf{B})$ th position in $\mathbf{B}$.

Then, for a channel $i \in \overline{\mathcal{A}}$, we have the following.

i) If $i \notin \mathcal{B}$, the free probability of channel $i$ is $\theta_{i}^{\text {free }}=\theta_{i}$. Therefore, the reward if user 1 selects channel $i$ in its $k$ th sensing is $G_{i}^{(1)}(k)=\theta_{i}^{\text {free }} U_{k}=\theta_{i} U_{k}$.

ii) If $i \in \mathcal{B}$, the free probability of channel $i$ is $\theta_{i}^{\text {free }}=\theta_{i}^{*}(1-$ $\left.\Pi_{j=1}^{g(i, \mathbf{B})-1}\left(1-\theta_{b_{j}}^{*}\right)\right)$, where the second term on the righthand side means the probability that user 2 does not proceed to sense channel $i$. Therefore, the reward if user 1 selects channel $i$ in its $k$ th sensing is

$$
G_{i}^{(1)}(k)=\theta_{i}^{\text {free }} U_{k}=\left[\theta_{i}^{*}\left(1-\Pi_{j=1}^{g(i, \mathbf{B})-1}\left(1-\theta_{b_{j}}^{*}\right)\right)\right] U_{k} .
$$

Then, the selection of the $k$ th sensing of user 1 is given by

$$
a_{k}=\arg \max _{i \in \overline{\mathcal{A}}} G_{i}^{(1)}(k)
$$

2) Selection of $b_{k}$ : To select $b_{k}$, the procedure is similar to that for $a_{k}$. The only difference lies in the reward of user 2 also selecting channel $a_{k}$ in its $k$ th sensing if $a_{k} \notin \mathcal{B}$. We have three possible situations, which are listed as follows.

i) FAIL_THEN_CONTINUE is adopted as the contentionresolution strategy: Similar to (3), we use the following equation to calculate the reward of user 2 selecting channel $a_{k}$ as its $k$ th channel to sense:

$$
G_{a_{k}}^{(2)}(k)=\theta_{a_{k}}^{*}\left(1-\Pi_{j=1}^{k-1}\left(1-\theta_{a_{j}}^{*}\right)\right) U_{k} .
$$

ii) FAIL_THEN_QUIT is adopted as the contention-resolution strategy: Consider the scenario when user 2 selects channel $a_{k}$ as its $k$ th channel to sense. If user 1 also proceeds to sense its $k$ th channel in its sensing order (i.e., $a_{k}$ ) and channel $a_{k}$ is primary-free, then user 1 and 2 will contend for the channel access, and thus, one will succeed and the other will quit subsequent channel sensings in the slot. Therefore, if user 2 also selects $a_{k}$ as its $k$ th channel to sense, compared with the case that user 2 selects another channel, user 2 will lose the chance to obtain rewards via sensing the subsequent channels (i.e., channels $\left.b_{k+1}, b_{k+2}, \ldots, b_{N}\right)$ in its sensing order. This loss of reward is approximated with an assumption that the subsequent channels in the sensing order of user 2 are in descending order of their free probabilities, i.e., $\theta_{b_{k+1}}^{\text {free }} \geq \theta_{b_{k+2}}^{\text {free }} \geq \cdots \geq \theta_{b_{N}}^{\text {free }}$. Then, the loss of reward is approximated by

$$
L\left(a_{k}\right)=\sum_{i=k+1}^{N}\left(\Pi_{j=k+1}^{i-1}\left(1-\theta_{b_{j}}^{\text {free }}\right)\right) \theta_{b_{i}}^{\text {free }} U_{i} .
$$

Therefore, the reward in (5) should be rewritten for user 2 selecting channel $a_{k}$ as its $k$ th channel to sense

$$
\begin{aligned}
G_{a_{k}}^{(2)}(k)=\theta_{a_{k}}^{*}[(1- & \left.\Pi_{j=1}^{k-1}\left(1-\theta_{a_{j}}^{*}\right)\right) U_{k} \\
& \left.-\left(\Pi_{j=1}^{k-1}\left(1-\theta_{a_{j}}^{*}\right)\right) L\left(a_{k}\right)\right]
\end{aligned}
$$

where the factor $\Pi_{j=1}^{k-1}\left(1-\theta_{a_{j}}^{*}\right)$ for $L\left(a_{k}\right)$ means the probability that user 1 does not stop at channel $a_{1}, a_{2}, \ldots$, or $a_{k-1}$.

iii) COLLIDE is adopted as the contention-resolution strategy: Consider the scenario where user 2 selects channel $a_{k}$ as its $k$ th channel to sense. If user 1 also proceeds to sense its $k$ th channel in its sensing order (i.e., $a_{k}$ ) and channel $a_{k}$ is primary-free, then a collision will happen. Therefore, if user 2 also selects $a_{k}$ as its $k$ th channel to sense, compared with the case that user 2 selects another channel, user 1 will lose its reward at channel $a_{k}$, and user 2 will lose the chance to obtain rewards via sensing the subsequent channels in its sensing order. Therefore, the loss of reward in (6) should be modified to

$$
L\left(a_{k}\right)=U_{k}+\sum_{i=k+1}^{N}\left(\Pi_{j=k+1}^{i-1}\left(1-\theta_{b_{j}}^{\text {free }}\right)\right) \theta_{b_{i}}^{\text {free }} U_{i}
$$

and (7) is still used to calculate the reward of user 2 selecting $a_{k}$ as its $k$ th channel to sense.

After $a_{k}$ and $b_{k}$ are selected, they are added into $\mathbf{A}, \mathbf{B}, \mathcal{A}$, and $\mathcal{B}$. Then, $\mathbf{A}=\left(a_{1}, a_{2}, \ldots, a_{k}\right), \mathbf{B}=\left(b_{1}, b_{2}, \ldots, b_{k}\right), \mathcal{A}=$ $\left\{a_{1}, a_{2}, \ldots, a_{k}\right\}$, and $\mathcal{B}=\left\{b_{1}, b_{2}, \ldots, b_{k}\right\}$. 


\section{Round-N Procedure}

In the last round, the only element in $\mathcal{N} \backslash \mathcal{A}$ is selected as $a_{N}$, and the only element in $\mathcal{N} \backslash \mathcal{B}$ is selected as $b_{N}$.

\section{Complexity of the Greedy Search Algorithm}

From the preceding procedure, it can be seen that the complexity of the greedy search algorithm is linear with the number of channels. Therefore, the complexity is $O(N)$, which is much less than the complexity in brute-force search.

\section{INCREMENTAL ALGORITHM}

It is observed in many scenarios that if we remove the channel with the smallest primary-free probability from an optimal sensing-order setting of the two users and keep other channels' relative positions in each sensing order unchanged, the remaining sensing-order setting is still optimal for the remaining channels. Accordingly, we propose a suboptimal algorithm, namely, the incremental algorithm, as follows. Here, "incremental" means that we gradually increase the size of the set of potential channels until the set size denoted by $k$ reaches $N$.

We start with $k=2$. The set of potential channels to be examined is $\{1,2\}$, since $\theta_{1}$ and $\theta_{2}$ are the two largest primary-free probabilities. By brute-force search, we can find an optimal sensing-order setting, i.e., $\mathbf{A}_{2}=\left(a_{1}, a_{2}\right)$ for user 1 and $\mathbf{B}_{2}=\left(b_{1}, b_{2}\right)$ for user 2 . Here, the subscript in $\mathbf{A}_{2}$ and $\mathbf{B}_{2}$ means the round index. The computation complexity of this round is $O\left(2^{2}\right)$.

In the round with $k=3$, from the previous round, we already know the sensing-order setting with two channels that have the two largest primary-free probabilities (i.e., channels 1 and 2). As an example, assume that $\mathbf{A}_{2}=(1,2)$ for user 1 and $\mathbf{B}_{2}=$ $(2,1)$ for user 2 . We need to add channel 3 into $\mathbf{A}_{2}$ and $\mathbf{B}_{2}$, while we keep the relative positions of channels 1 and 2 in $\mathbf{A}_{2}$ and $\mathbf{B}_{2}$ unchanged. After channel 3 is added, the new sensing orders are $\mathbf{A}^{\prime}$ and $\mathbf{B}^{\prime}$ for users 1 and 2, respectively. $\mathbf{A}^{\prime}$ has three possible cases, i.e., $(3,1,2),(1,3,2)$, and $(1,2,3)$, while $\mathbf{B}^{\prime}$ also has three possible cases, i.e., $(3,2,1),(2,3,1)$, and $(2,1,3)$. Therefore, we have $3^{2}=9$ possible combinations of $\left(\mathbf{A}^{\prime}, \mathbf{B}^{\prime}\right)$. Among them, the combination with the maximal average system throughput is selected as $\left(\mathbf{A}_{3}, \mathbf{B}_{3}\right)$. The computational complexity in this round is $O\left(3^{2}\right)$.

In the round with $k \in\{4, \ldots, N\}$, from the previous round, we already know the sensing-order setting with $k-1$ channels that have the $k-1$ largest primary-free probabilities, which is denoted by $\mathbf{A}_{k-1}=\left(a_{1}, a_{2}, \ldots, a_{k-1}\right)$ for user 1 and by $\mathbf{B}_{k-1}=\left(b_{1}, b_{2}, \ldots, b_{k-1}\right)$ for user 2. Both $\mathbf{A}_{k-1}$ and $\mathbf{B}_{k-1}$ are permutations of $(1,2, \ldots, k-1)$. We need to add channel $k$ into $\mathbf{A}_{k-1}$ and $\mathbf{B}_{k-1}$, while keeping the relative positions of existing elements in $\mathbf{A}_{k-1}$ and $\mathbf{B}_{k-1}$ unchanged. After channel $k$ is added, the new sensing orders are $\mathbf{A}^{\prime}$ and $\mathbf{B}^{\prime}$ for users 1 and 2, respectively. Either $\mathbf{A}^{\prime}$ or $\mathbf{B}^{\prime}$ has $k$ possible cases. Then, we have $k^{2}$ possible combinations of $\left(\mathbf{A}^{\prime}, \mathbf{B}^{\prime}\right)$. Among them, the combination with the maximal average system throughput is selected as $\left(\mathbf{A}_{k}, \mathbf{B}_{k}\right)$. The computational complexity in this round is $O\left(k^{2}\right)$.
The aforementioned procedure is repeated until $k=N$. Then, $\mathbf{A}_{N}$ and $\mathbf{B}_{N}$ are the sensing orders of the two users, with $N$ potential channels.

The computational complexity of the incremental algorithm is thus $O\left(\sum_{i=2}^{N} i^{2}\right)$, which is much less than the complexity of brute-force search.

\section{NUMERICAL RESUlTS}

To evaluate the performance of the proposed greedy search and incremental algorithms, we consider $N=7$ potential channels, with each channel sensing time being equal to $\tau=0.1$. The primary-free probability of channel $i$ (i.e., $\theta_{i}$ ) is given by $\theta_{i}=0.05 \cdot \kappa_{i}$, where $\kappa_{i}$ is an integer. We get the numerical results for the following three tests.

1) Test I: This test includes all the scenarios with $\kappa_{i}$ 's being even integers, $2 \leq \kappa_{1} \leq 18,2 \leq \kappa_{2} \leq \kappa_{1}, 2 \leq \kappa_{3} \leq \kappa_{2}$, and $0 \leq \kappa_{i} \leq \kappa_{i-1}$ for $i=4,5,6$, and 7 . Note that, in this test, all $\theta_{i}$ 's are within [0,0.9].

2) Test II: This test includes all the scenarios with $10 \leq \kappa_{i} \leq$ 18 and $\kappa_{1} \geq \kappa_{2} \geq \cdots \geq \kappa_{7}$. Note that, in this test, all $\theta_{i}$ 's are within $[0.5,0.9]$.

3) Test III: This test includes all the scenarios with $1 \leq \kappa_{i} \leq$ 10 and $\kappa_{1} \geq \kappa_{2} \geq \cdots \geq \kappa_{7}$. Note that, in this test, all $\theta_{i}$ 's are within $[0.05,0.5]$.

In each scenario of each test, we obtain the maximal average system throughput through brute-force search (for an optimal sensing-order setting), which is denoted by $T_{\text {opt }}$, and the average system throughput of the greedy search and incremental algorithms, which is denoted by $T_{\text {greedy }}$ and $T_{\text {incremental }}$, respectively. We also obtain the relative difference of the average system throughput of the greedy search and incremental algorithms from the maximal average system throughput, which is defined as $\left(T_{\text {opt }}-T_{\text {greedy }}\right) /\left(T_{\text {opt }}\right)$ and $\left(T_{\text {opt }}-\right.$ $\left.T_{\text {incremental }}\right) /\left(T_{\text {opt }}\right)$, respectively. It is observed that all the relative difference values in all scenarios are below 5\%. Table I shows the percentage of the relative difference values being in different intervals and the mean and standard deviation (Std) of the relative difference values when the contention-resolution strategy is FAIL_THEN_CONTINUE, FAIL_THEN_QUIT, or COLLIDE. It can be seen that, with FAIL_THEN_CONTINUE, the greedy search algorithm is very likely (with probability $93.02 \%$ in Test I, $71.62 \%$ in Test II, and $63.91 \%$ in Test III) to achieve an optimal solution, while the likelihood is decreased with FAIL_THEN_QUIT or COLLIDE. This is because of the approximation used to estimate the loss of reward in a contention. On the other hand, the incremental algorithm with FAIL_THEN_CONTINUE can achieve an optimal solution with probability $99.64 \%$ in Test I, $97.31 \%$ in Test II, and $91.79 \%$ in Test III. The likelihood is slightly decreased when FAIL_THEN_QUIT is the contention-resolution strategy. This is because, in each round of the incremental algorithm, the relative positions of the channels determined in prior rounds do not change. Therefore, it is likely that a channel may have the same sensing position in the two users' sensing orders. Thus, a user will fail in the contention and lose the reward of subsequent sensings if both users simultaneously proceed to sense the channel and the channel is primary-free. The reward 
TABLE I

Percentage of the Relative Difference Values and the Mean and Std

\begin{tabular}{|c|c|c|c|c|c|c|c|c|}
\hline Test & \multicolumn{2}{|c|}{ Relative difference } & 0 & $(0,0.1 \%]$ & $(0.1 \%, 1 \%]$ & $(1 \%, 5 \%]$ & Mean & Std \\
\hline \multirow{6}{*}{ I } & FAIL_THEN & Greedy Search & $93.02 \%$ & $6.73 \%$ & $0.25 \%$ & 0 & $0.0015 \%$ & $0.0097 \%$ \\
\hline & _CONTINUE & Incremental & $99.64 \%$ & $0.36 \%$ & 0 & 0 & $0.000037 \%$ & $0.0008 \%$ \\
\hline & FAIL_THEN & Greedy Search & $64.47 \%$ & $21.55 \%$ & $13.47 \%$ & $0.50 \%$ & $0.0541 \%$ & $0.1565 \%$ \\
\hline & _QUIT & Incremental & $88.86 \%$ & $10.87 \%$ & $0.26 \%$ & 0 & $0.0022 \%$ & $0.0112 \%$ \\
\hline & COIJTDF & Greedy Search & $51.33 \%$ & $33.23 \%$ & $13.63 \%$ & $1.81 \%$ & $0.0881 \%$ & $0.2810 \%$ \\
\hline & & Incremental & $16.50 \%$ & $28.63 \%$ & $54.59 \%$ & $0.29 \%$ & $0.1733 \%$ & $0.1873 \%$ \\
\hline \multirow{6}{*}{ II } & FAIL_THEN & Greedy Search & $71.62 \%$ & $28.38 \%$ & 0 & 0 & $0.0005 \%$ & $0.0016 \%$ \\
\hline & _CONTINUE & Incremental & $97.31 \%$ & $2.69 \%$ & 0 & 0 & $0.000062 \%$ & $0.00064 \%$ \\
\hline & FAIL_THEN & Greedy Search & $53.55 \%$ & $42.88 \%$ & $3.57 \%$ & 0 & $0.014 \%$ & $0.056 \%$ \\
\hline & -QUIT & Incremental & $85.67 \%$ & $14.33 \%$ & 0 & 0 & $0.0007 \%$ & $0.0027 \%$ \\
\hline & COLITDF & Greedy Search & $47.94 \%$ & $50.67 \%$ & $1.37 \%$ & $0.02 \%$ & $0.0096 \%$ & $0.051 \%$ \\
\hline & COLLIDE & Incremental & $10.97 \%$ & $87.85 \%$ & $1.18 \%$ & 0 & $0.022 \%$ & $0.023 \%$ \\
\hline \multirow{6}{*}{ III } & FAIL_THEN & Greedy Search & $63.91 \%$ & $32.93 \%$ & $3.16 \%$ & 0 & $0.015 \%$ & $0.031 \%$ \\
\hline & _CONTINUE & Incremental & $91.79 \%$ & $7.80 \%$ & $0.41 \%$ & 0 & $0.0024 \%$ & $0.013 \%$ \\
\hline & FAIL_THEN & Greedy Search & $54.37 \%$ & $33.88 \%$ & $11.09 \%$ & $0.66 \%$ & $0.045 \%$ & $0.14 \%$ \\
\hline & _QUIT & Incremental & $84.49 \%$ & $12.19 \%$ & $3.32 \%$ & 0 & $0.0088 \%$ & $0.029 \%$ \\
\hline & COLLTDE & Greedy Search & $37.99 \%$ & $40.78 \%$ & $21.23 \%$ & 0 & $0.057 \%$ & $0.081 \%$ \\
\hline & & Incremental & $0.48 \%$ & $1.86 \%$ & $94.95 \%$ & $2.71 \%$ & $0.42 \%$ & $0.23 \%$ \\
\hline
\end{tabular}

loss determines that the incremental algorithm may not always be optimal. When COLLIDE is adopted for the incremental algorithm, the loss of reward is much more severe, and thus, the incremental algorithm only has a small probability to achieve an optimal solution. However, since almost all the relative difference values in all the scenarios are below $1 \%$, the performance of the incremental algorithm is still promising when COLLIDE is the contention-resolution strategy.

In summary, generally, both algorithms work very well with all the three contention-resolution strategies. As a comparison, the greedy search algorithm has much lower computational complexity. The incremental algorithm can achieve slightly larger system throughput with FAIL_THEN_CONTINUE and FAIL_THEN_QUIT, while the greedy search algorithm leads to better throughput performance with COLLIDE.

\section{IMPACT OF ADAPTIVE MOdULATION}

If a secondary user knows its channel quality (i.e., SNR) to its receiver, adaptive modulation can be adopted to utilize the time-varying feature of the channels. The secondary user can adapt its transmission rate according to the channel quality. For channel $i$, if a user senses it free and the SNR of the user is $\mathrm{SNR}_{i}$, then the achievable transmission rate of the user is $f\left(\mathrm{SNR}_{i}\right)$. Here, $f(\cdot)$ is a nondescending function mapping the SNR to the transmission rate.

By adaptive modulation, a secondary user can opportunistically select a free channel with good channel quality to transmit, which is referred to as multichannel diversity. In multichannel diversity, additional overhead is needed to estimate the channel gain, e.g., small control packets may be sent between the sender and receiver. Despite the overhead, it is shown in [25] that multichannel diversity can result in an overall benefit. In the open literature, multichannel diversity has been well exploited in cognitive-radio-related research efforts [14][16], [26].

In Section VII-D, a scheme will be given for a secondary user to estimate its channel SNR to its receiver. With adaptive modulation, when a user senses a free channel with poor channel quality, it may skip this channel, with the expectation that it may have better quality in other channels [16], [26]. To determine when a user should stop at a channel (i.e., the channel is free and has a satisfactory SNR), similar to the research in [16] and [26], an SNR threshold is assigned for the channel. When the user proceeds to sense the channel, if the channel is free and the channel SNR is above the threshold, the user will stop at the channel (i.e., start its contention in the channel); otherwise, the user will proceed to sense the next channel in its sensing order. Therefore, the configuration of each user includes a sensing order and an SNR threshold for each channel in its sensing order. For user 1, its sensing order is $\left(a_{1}, a_{2}, \ldots, a_{N}\right)$ with SNR thresholds $\left(\Gamma_{a_{1}}^{(1)}, \Gamma_{a_{2}}^{(1)}, \ldots, \Gamma_{a_{N}}^{(1)}\right)$. For user 2 , its sensing order is $\left(b_{1}, b_{2}, \ldots, b_{N}\right)$ with SNR thresholds $\left(\Gamma_{b_{1}}^{(2)}, \Gamma_{b_{2}}^{(2)}, \ldots, \Gamma_{b_{N}}^{(2)}\right)$.

To find an optimal system configuration, two questions need to be answered: 1) what is the optimal stopping rule, that is, how should a user determine the SNR threshold of a channel in its sensing order? 2) Does the adoption of adaptive modulation affect the optimal sensing-order setting of the two users (compared with the case without adaptive modulation)? The two questions are investigated as follows.

\section{A. Stopping Rule}

Consider that a user proceeds to sense its $k$ th channel in its sensing order, e.g., channel $i$, which is free. If the user has an SNR value denoted by $\mathrm{SNR}_{i}$, its instantaneous reward at the channel is $c_{k} f\left(\mathrm{SNR}_{i}\right)$ times the probability that it will win the contention (if any) in the channel. Here, $c_{k}$ is given in (1). The user also has an expected reward if it skips channel $i$ and proceeds to sense the next channel in its sensing order. It may seem plausible and intuitive that the user should stop at channel $i$ if the instantaneous reward of the channel is larger than the expected reward of skipping the channel. This stopping rule is referred to as the traditional stopping rule in the following.

Next, we investigate whether the traditional stopping rule is optimal in a single-user case and a two-user case, respectively.

1) Optimality of the Traditional Stopping Rule in a SingleUser Case: The traditional stopping rule has been used in single-user multichannel medium access control in the 
literature [16], [26]. Here, we prove the optimality of the traditional stopping rule in the single-user case.

Consider a (secondary) user with $N$ potential channels. Channel $i$ is primary-free with probability $\theta_{i}$. The probability density function (pdf) of channel $i$ 's SNR is denoted by $h_{i}(\gamma)$ for SNR value $\gamma$. The SNR of each channel is independent of any other channel. The user's sensing order is $\left(a_{1}, a_{2}, \ldots, a_{N}\right)$, which is a permutation of $(1,2, \ldots, N)$. For channel $a_{i}$, the SNR threshold is $\Gamma_{a_{i}}$. Particularly, for the last channel in the sensing order, the SNR threshold is zero, i.e., $\Gamma_{a_{N}}=0$. If the user proceeds to sense the $k$ th channel in the sensing order, i.e., $a_{k}$, the user will transmit at the channel if $\mathrm{SNR}_{a_{k}} \geq \Gamma_{a_{k}}$, where $\mathrm{SNR}_{a_{k}}$ is the instantaneous SNR in channel $a_{k}$. No recall is allowed. This means that if the user skips a primary-free channel, it is not allowed to return to transmit at that channel.

If the user proceeds to sense channel $a_{N}$, its instantaneous reward if channel $a_{N}$ is primary-free is given by $w_{a_{N}}=$ $c_{N} f\left(\mathrm{SNR}_{a_{N}}\right)$. Furthermore, the reward expectation of sensing channel $a_{N}$ is $W_{a_{N}}=\theta_{a_{N}} \mathbb{E}\left[w_{a_{N}}\right]$, where $\mathbb{E}[\cdot]$ denotes expectation.

When the user proceeds to sense its $i$ th $(i \leq N-1)$ channel in its sensing order, i.e., channel $a_{i}$, its reward (if the channel is primary-free) is

$$
w_{a_{i}}=\left\{\begin{array}{lc}
c_{i} f\left(\mathrm{SNR}_{a_{i}}\right), & \text { if } \mathrm{SNR}_{a_{i}}>\Gamma_{a_{i}} \\
& \left(\text { stop at channel } a_{i}\right) \\
W_{a_{i+1}}, & \text { otherwise (proceed to sense } \\
& \text { channel } \left.a_{i+1}\right)
\end{array}\right.
$$

where $W_{a_{i+1}}(i \leq N-1)$ is the expected reward if the user proceeds to sense channel $a_{i+1}$, which is given by

$$
W_{a_{i+1}}= \begin{cases}\theta_{a_{i+1}} \mathbb{E}\left[w_{a_{i+1}}\right]+\left(1-\theta_{a_{i+1}}\right) W_{a_{i+2}}, & \text { if } i<N-1 \\ \theta_{a_{i+1}} \mathbb{E}\left[w_{a_{i+1}}\right], & \text { if } i=N-1 .\end{cases}
$$

From (9) and (10), we can recursively obtain the values of all $W_{a_{i}}$ 's, starting from $W_{a_{N}}$ until $W_{a_{1}}$ [16], [26]. $W_{a_{1}}$ is the average throughput of the user with the $N$ potential channels.

Lemma 1: With a specific sensing order $\left(a_{1}, a_{2}, \ldots, a_{N}\right)$, the traditional stopping rule is optimal. This means that the optimal threshold setting $\left(\Gamma_{a_{1}}, \Gamma_{a_{2}}, \ldots, \Gamma_{a_{N}}\right)$ satisfies $c_{i} f\left(\Gamma_{a_{i}}\right)=$ $W_{a_{i+1}}, 1 \leq i \leq N-1$ and $\Gamma_{a_{N}}=0$.

Proof: If the user proceeds to sense its last channel in its sensing order, i.e., $a_{N}$, it should transmit if the channel is sensed to be free. Therefore, $\Gamma_{a_{N}}=0$.

From (9) and (10), $\forall i \leq N-1$, we have

$$
\begin{aligned}
W_{a_{1}}= & \left\{\sum_{l=1}^{i}\left[\Pi_{k=1}^{l-1}\left(1-\theta_{a_{k}} \int_{\Gamma_{a_{k}}}^{\infty} h_{a_{k}}(\gamma) d \gamma\right)\right] \theta_{a_{l}}\right. \\
& \left.\cdot c_{l} \int_{\Gamma_{a_{l}}}^{\infty} f(\gamma) h_{a_{l}}(\gamma) d \gamma\right\} \\
+ & {\left[\Pi_{k=1}^{i}\left(1-\theta_{a_{k}} \int_{\Gamma_{a_{k}}}^{\infty} h_{a_{k}}(\gamma) d \gamma\right)\right] \cdot W_{a_{i+1}} }
\end{aligned}
$$

To achieve the optimal $W_{a_{1}}$ value, we should have $\left(\partial W_{a_{1}} / \partial \Gamma_{a_{i}}\right)=0$. We get

$$
\begin{array}{r}
{\left[\prod_{k=1}^{i-1}\left(1-\theta_{a_{k}} \int_{\Gamma_{a_{k}}}^{\infty} h_{a_{k}}(\gamma) d \gamma\right)\right]} \\
\cdot\left[\theta_{a_{i}} c_{i} f\left(\Gamma_{a_{i}}\right) h_{a_{i}}\left(\Gamma_{a_{i}}\right)-\theta_{a_{i}} h_{a_{i}}\left(\Gamma_{a_{i}}\right) W_{a_{i+1}}\right]=0
\end{array}
$$

and furthermore, we have $c_{i} f\left(\Gamma_{a_{i}}\right)=W_{a_{i+1}}, 1 \leq i \leq N-1$. This completes the proof.

2) Stopping Rule for a Two-User Case: When two users, i.e., users 1 and 2, are considered, the traditional stopping rule may no longer be optimal. We use the following example to show the difference of the traditional stopping rule from an optimal stopping rule for a two-user case.

Consider the situation with two channels, i.e., channels 1 and 2 , with primary-free probabilities $\theta_{1}>0$ and $\theta_{2}>0$. The pdf of user $i$ 's SNR in channel $j$ is $h_{j}^{(i)}(\gamma)>0$, for $i, j \in\{1,2\}, \gamma \in(0, \infty)$. User 1 has sensing order $(1,2)$ and associated SNR thresholds $\left(\Gamma_{1}^{(1)}, \Gamma_{2}^{(1)}\right)$. User 2 has sensing order $(2,1)$ and associated SNR thresholds $\left(\Gamma_{2}^{(2)}, \Gamma_{1}^{(2)}\right)$. If user 1 proceeds to channel 2 and senses it to be free (which means that channel 2 is primary-free and user 2 does not stop at channel 2 in its first sensing), user 1 should transmit in channel 2 with whatever SNR. Thus, $\Gamma_{2}^{(1)}=0$. Similarly, we have $\Gamma_{1}^{(2)}=0$. Therefore, we only need to determine $\Gamma_{1}^{(1)}$ and $\Gamma_{2}^{(2)}$

Traditional stopping rule: If user 1 proceeds to sense channel 2, it can transmit only when channel 2 is primary-free and user 2 does not stop at channel 2 at its first sensing. Thus, the reward expectation of user 1 proceeding to sense channel 2 is

$$
W_{2}^{(1)}=\theta_{2} \cdot \int_{0}^{\Gamma_{2}^{(2)}} h_{2}^{(2)}(\gamma) d \gamma \cdot c_{2} \int_{0}^{\infty} f(\gamma) h_{2}^{(1)}(\gamma) d \gamma
$$

where the second term on the right-hand side is the probability that user 2 does not stop at channel 2 at its first sensing. Therefore, according to the traditional stopping rule, we have

$$
\begin{aligned}
c_{1} f\left(\Gamma_{1}^{(1)}\right) & =W_{2}^{(1)} \\
& =\theta_{2} \cdot \int_{0}^{\Gamma_{2}^{(2)}} h_{2}^{(2)}(\gamma) d \gamma \cdot c_{2} \int_{0}^{\infty} f(\gamma) h_{2}^{(1)}(\gamma) d \gamma
\end{aligned}
$$

Similarly, we have

$$
\begin{aligned}
c_{1} f\left(\Gamma_{2}^{(2)}\right) & =W_{1}^{(2)} \\
& =\theta_{1} \cdot \int_{0}^{\Gamma_{1}^{(1)}} h_{1}^{(1)}(\gamma) d \gamma \cdot c_{2} \int_{0}^{\infty} f(\gamma) h_{1}^{(2)}(\gamma) d \gamma
\end{aligned}
$$


Optimal stopping rule: For user 1, its throughput is given by

$$
\begin{array}{r}
W_{1}^{(1)}=\theta_{1} c_{1} \int_{\Gamma_{1}^{(1)}}^{\infty} f(\gamma) h_{1}^{(1)}(\gamma) d \gamma+\left(1-\theta_{1} \int_{\Gamma_{1}^{(1)}}^{\infty} h_{1}^{(1)}(\gamma) d \gamma\right. \\
\cdot \theta_{2} \cdot \int_{0}^{\Gamma_{2}^{(2)}} h_{2}^{(2)}(\gamma) d \gamma \cdot c_{2} \int_{0}^{\infty} f(\gamma) h_{2}^{(1)}(\gamma) d \gamma .
\end{array}
$$

For user 2, its throughput is given by

$$
\begin{array}{r}
W_{2}^{(2)}=\theta_{2} c_{1} \int_{\Gamma_{2}^{(2)}}^{\infty} f(\gamma) h_{2}^{(2)}(\gamma) d \gamma+\left(1-\theta_{2} \int_{\Gamma_{2}^{(2)}}^{\infty} h_{2}^{(2)}(\gamma) d \gamma\right) \\
\cdot \theta_{1} \cdot \int_{0}^{\Gamma_{1}^{(1)}} h_{1}^{(1)}(\gamma) d \gamma \cdot c_{2} \int_{0}^{\infty} f(\gamma) h_{1}^{(2)}(\gamma) d \gamma
\end{array}
$$

To achieve the optimal system throughput, we should have

$$
\begin{aligned}
& \frac{\partial\left(W_{1}^{(1)}+W_{2}^{(2)}\right)}{\partial \Gamma_{1}^{(1)}}=0 \\
& \frac{\partial\left(W_{1}^{(1)}+W_{2}^{(2)}\right)}{\partial \Gamma_{2}^{(2)}}=0 .
\end{aligned}
$$

Then, we get

$$
\begin{aligned}
c_{1} f\left(\Gamma_{1}^{(1)}\right)= & \theta_{2} \cdot \int_{0}^{\Gamma_{2}^{(2)}} h_{2}^{(2)}(\gamma) d \gamma \cdot c_{2} \int_{0}^{\infty} f(\gamma) h_{2}^{(1)}(\gamma) d \gamma \\
& +\left(1-\theta_{2} \int_{\Gamma_{2}^{(2)}}^{\infty} h_{2}^{(2)}(\gamma) d \gamma\right) c_{2} \int_{0}^{\infty} f(\gamma) h_{1}^{(2)}(\gamma) d \gamma \\
c_{1} f\left(\Gamma_{2}^{(2)}\right)= & \theta_{1} \cdot \int_{0}^{\Gamma_{1}^{(1)}} h_{1}^{(1)}(\gamma) d \gamma \cdot c_{2} \int_{0}^{\infty} f(\gamma) h_{1}^{(2)}(\gamma) d \gamma \\
& +\left(1-\theta_{1} \int_{\Gamma_{1}^{(1)}}^{\infty} h_{1}^{(1)}(\gamma) d \gamma\right) c_{2} \int_{0}^{\infty} f(\gamma) h_{2}^{(1)}(\gamma) d \gamma
\end{aligned}
$$

which are apparently different from (13) and (14) for the traditional stopping rule.

\section{B. Impact of Adaptive Modulation on the Optimal Sensing-Order Setting}

The answer to the question of whether the adoption of adaptive modulation affects the optimal sensing-order setting of the two users is essential. This is because if the answer is "no," then the derivation of an optimal system configuration (including the sensing-order setting and the SNR threshold setting) with adaptive modulation can be divided into two steps: one step to find an optimal sensing-order setting without adaptive modulation and the other step to select an optimal SNR threshold setting when adaptive modulation is adopted. However, unfortunately, the answer to that question is "yes." We use the following example to demonstrate that an optimal sensing-order setting without adaptive modulation may not be optimal when adaptive modulation is adopted.

Consider two users, i.e., users 1 and 2, with two channels, i.e., channels 1 and 2 . The primary-free probabilities of the two channels are $\theta_{1} \gg 0$ and $\theta_{2}=0^{+}$, respectively. Here, $0^{+}$ means an infinitely small positive value. The pdf of the SNR of each user in each channel is common, which is denoted by $h(\gamma)$. FAIL_THEN_QUIT is the contention-resolution strategy. Therefore, when adaptive modulation is not adopted, the optimal sensing-order setting is $(1,2)$ for user 1 and $(2,1)$ for user 2, which is referred to as the DIFF_ORDER setting. When adaptive modulation is adopted with the sensing-order setting being the DIFF_ORDER setting, we denote the optimal SNR threshold setting as $\left(\Gamma_{1}^{(1)}, \Gamma_{2}^{(1)}\right)$ for user 1 and $\left(\Gamma_{2}^{(2)}, \Gamma_{1}^{(2)}\right)$ for user 2 . Since channel 2 is primary-free with probability $0^{+}$, either user's SNR threshold in channel 2 is set to be zero. Furthermore, when user 2 proceeds to sense channel 1 in its second sensing, its SNR threshold should be zero because if user 2 senses channel 1 to be free, channel 1 should be primaryfree, and user 1 should have skipped channel 1 in its first sensing due to an unsatisfactory SNR. Therefore, the optimal SNR threshold setting is $\left(\Gamma_{1}^{(1)}, 0\right)$ for user 1 and $(0,0)$ for user 2 . We have the following lemma.

Lemma 2: $c_{1} f\left(\Gamma_{1}^{(1)}\right)=c_{2} \int_{0}^{\infty} f(\gamma) h(\gamma) d \gamma$.

Proof: The system throughput is given by

$$
\begin{aligned}
T_{\mathrm{DIFF}}=\theta_{1} \cdot c_{1} \int_{\Gamma_{1}^{(1)}}^{\infty} f(\gamma) h(\gamma) d \gamma & \\
& +\theta_{1} \cdot \int_{0}^{\Gamma_{1}^{(1)}} h(\gamma) d \gamma \cdot c_{2} \int_{0}^{\infty} f(\gamma) h(\gamma) d \gamma
\end{aligned}
$$

On the right-hand side of (15), the first term means that user 1 transmits in channel 1 upon its first sensing if channel 1 is primary-free and user 1's SNR in channel 1 is above the threshold, and the second term means that user 2 transmits in channel 1 upon its second sensing if channel 1 is primary-free and user 1 skips channel 1 in its first sensing.

For the optimal SNR threshold setting, we have

$$
\frac{d T_{\mathrm{DIFF}}}{d \Gamma_{1}^{(1)}}=0
$$

which can lead to

$$
c_{1} f\left(\Gamma_{1}^{(1)}\right)=c_{2} \int_{0}^{\infty} f(\gamma) h(\gamma) d \gamma
$$

This completes the proof. 
Lemma 3: The DIFF_ORDER setting is not optimal if $c_{1} \int_{\Gamma_{1}^{(1)}}^{\infty} f(\gamma) h(\gamma) d \gamma>c_{2} \int_{0}^{\infty} f(\gamma) h(\gamma) d \gamma$.

Proof: We consider a new sensing-order setting where both users 1 and 2 use $(1,2)$ as the sensing order, which is referred to as the SAME_ORDER setting. With the SAME_ORDER setting, we let the SNR threshold setting be $\left(\Gamma_{1}^{(1)}, 0\right)$ for both users, where $\Gamma_{1}^{(1)}$ is user 1's optimal SNR threshold in channel 1 with the DIFF_ORDER setting (as given in Lemma 2).

The system throughput of the SAME_ORDER setting is

$$
\begin{aligned}
T_{\text {SAME }}=\theta_{1}\left(c_{1} \int_{\Gamma_{1}^{(1)}}^{\infty} h(\gamma) d \gamma \int_{\Gamma_{1}^{(1)}}^{\infty} f(\gamma) h(\gamma) d \gamma\right. \\
\left.+2 \cdot c_{1} \int_{0}^{\Gamma_{1}^{(1)}} h(\gamma) d \gamma \int_{\Gamma_{1}^{(1)}}^{\infty} f(\gamma) h(\gamma) d \gamma\right) .
\end{aligned}
$$

On the right-hand side of (16), the first term in the bracket means that both users have an SNR in channel 1 above the threshold (i.e., one user wins the contention and transmits, and the other user fails and quits), and the second term means that one user has an SNR in channel 1 above the threshold (i.e., stops at channel 1), while the other user has an SNR in channel 1 below the threshold (i.e., skips channel 1 and proceeds to the next channel). After some mathematical manipulation, we have

$$
\begin{aligned}
T_{\mathrm{SAME}}=\theta_{1} \cdot c_{1} & \int_{\Gamma_{1}^{(1)}}^{\infty} f(\gamma) h(\gamma) d \gamma \\
& +\theta_{1} \cdot c_{1} \int_{0}^{\Gamma_{1}^{(1)}} h(\gamma) d \gamma \int_{\Gamma_{1}^{(1)}}^{\infty} f(\gamma) h(\gamma) d \gamma .
\end{aligned}
$$

From (15) and (17), we have

$$
\begin{aligned}
T_{\mathrm{SAME}}-T_{\mathrm{DIFF}} & =\theta_{1} \int_{0}^{\Gamma_{1}^{(1)}} h(\gamma) d \gamma \\
& \cdot\left(c_{1} \int_{\Gamma_{1}^{(1)}}^{\infty} f(\gamma) h(\gamma) d \gamma-c_{2} \int_{0}^{\infty} f(\gamma) h(\gamma) d \gamma\right)
\end{aligned}
$$

which means that if $c_{1} \int_{\Gamma_{1}^{(1)}}^{\infty} f(\gamma) h(\gamma) d \gamma>c_{2} \int_{0}^{\infty} f(\gamma) h(\gamma) d \gamma$, we have $T_{\mathrm{SAME}}>T_{\mathrm{DIFF}}$ (i.e., the DIFF_ORDER setting is not optimal). This completes the proof.

Consider the Shannon's channel capacity as the achievable transmission rate, i.e., $f(\gamma)=\log (1+\gamma)$, and Rayleigh fading channels, i.e., $h(\gamma)=(1 / \Gamma) e^{-(\gamma / \Gamma)}$ (where $\Gamma=10$ is the average SNR in a channel as an example). Table II shows the ratio of $c_{1} \int_{\Gamma_{1}^{(1)}}^{\infty} f(\gamma) h(\gamma) d \gamma$ to $c_{2} \int_{0}^{\infty} f(\gamma) h(\gamma) d \gamma$ for different values of channel-sensing time $\tau$. It can be seen that, when $\tau \geq 0.15$, we have $c_{1} \int_{\Gamma_{1}^{(1)}}^{\infty} f(\gamma) h(\gamma) d \gamma>c_{2} \int_{0}^{\infty} f(\gamma) h(\gamma) d \gamma$, and thus,
TABLE II

RATIO OF $c_{1} \int_{\Gamma_{1}^{(1)}}^{\infty} f(\gamma) h(\gamma) d \gamma$ TO $c_{2} \int_{0}^{\infty} f(\gamma) h(\gamma) d \gamma$

\begin{tabular}{c|c|c|c|c|c|c|c|c|c}
\hline \hline$\tau$ & 0.05 & 0.1 & 0.15 & 0.2 & 0.25 & 0.3 & 0.35 & 0.4 & 0.45 \\
\hline Ratio & 0.79 & 0.89 & 1.01 & 1.16 & 1.36 & 1.64 & 2.09 & 2.95 & 5.48 \\
\hline \hline
\end{tabular}

the SAME_ORDER setting is the optimal sensing-order setting.

\section{Discussion}

In the preceding sections, we have two observations for a two-user cognitive radio network: 1) When the sensing-order setting of the two users is fixed, the traditional stopping rule may not lead to an optimal SNR threshold setting, and 2) the adoption of adaptive modulation may affect the optimal sensing-order setting of the two users. These observations imply that the sensing-order setting and the SNR threshold setting should be jointly determined from a systematic viewpoint to achieve the maximal system throughput. Although, in this paper, a detailed algorithm to derive the optimal system configuration with adaptive modulation is not given, the two observations should be able to pave the way for further research efforts.

\section{Implementation Issues}

To achieve adaptive modulation in cognitive radio networks, two challenges are experienced: the hidden-terminal problem and the channel-SNR-estimation problem.

i) Hidden-terminal problem: Consider a scenario with a primary transmitter and its receiver and a secondary transmitter and its receiver. The primary transmitter is active, and its location is far away from the secondary transmitter but close to the secondary receiver. Then, the secondary transmitter may not sense the signal from the primary transmitter and, thus, transmits its own signal. However, the secondary receiver cannot successfully receive this signal due to strong interference from the primary transmitter. This is the well-known hidden-terminal problem.

ii) Channel-SNR-estimation problem: In adaptive modulation, the secondary transmitter needs to know the channel SNR to its receiver before its information transmission.

To solve the preceding problems, we propose to let the secondary receiver instead of the secondary transmitter perform the sensing task. Furthermore, in the time slot structure in Fig. 1, a probing time with duration $\Delta$ is inserted after each sensing duration $\tau$. It might be difficult to give a specific value of $\Delta$, since the value should depend on the channel bandwidth, acquisition technique, modulation scheme, etc. In general, an appropriate $\Delta$ value should ensure that the transmission phase still occupies a significant portion in a time slot.

At the beginning of each time slot, the secondary receiver first senses its first channel (in its sensing order) for a duration of $\tau$.

i) If the channel is sensed to be free, the secondary receiver transmits a probe in the following probing duration $\Delta$. The probe is transmitted using spread spectrum technology. Specifically, each secondary transmitter is 
assigned a unique spreading code. The probe sent from the secondary receiver is spread by the spreading code of its secondary transmitter. The secondary transmitter keeps scanning its own spreading code and can thus successfully receive the probe. Through the reception of the probe, the secondary transmitter can estimate the channel SNR from the secondary receiver to itself. This SNR is also the estimated channel SNR from the secondary transmitter to the secondary receiver, as channel reciprocity is assumed here.

ii) If the channel is sensed to be busy, the secondary receiver skips the following probing time $\Delta$ and then starts to sense its next channel in its sensing order.

The aforementioned procedure is repeated in the sensing phase of each slot.

As the sensing task is performed by the secondary receiver, the hidden-terminal problem does not exist. Furthermore, through the channel probing from the secondary receiver, the channel SNR can be estimated at the secondary transmitter side.

\section{CONCLUSion AND Further Discussions}

In cognitive radio networks, one objective is to achieve the maximal spectrum efficiency. In this paper, we have demonstrated our research efforts to achieve the maximal spectrum efficiency through the selecting of sensing orders in a two-user cognitive radio network. For the case without adaptive modulation, low-complexity algorithms have been proposed that have comparable performance with that of an optimal solution and particularly have a high probability to reach an optimal point when a backoff mechanism is used. For the case with adaptive modulation, our observations imply that the sensingorder setting and stopping rule should be jointly designed from a systematic point of view.

Sensing is assumed to be perfect in this paper. In reality, sensing errors are inevitable. A channel-sensing error is either a missed detection (i.e., the channel is occupied by primary users, but the secondary user senses it to be idle) or a false alarm (i.e., the channel is idle, but the secondary user senses it to be busy). Let $P_{m}$ and $P_{f}$ denote the missed-detection probability and false-alarm probability, respectively. Then, the probability that a secondary user senses channel $i$ to be idle is given by $\theta_{i}\left(1-P_{f}\right)+\left(1-\theta_{i}\right) P_{m}$. If a secondary user senses an idle channel and transmits in the channel, its probability of successful transmission (i.e., not colliding with possible primary activities $)$ is $\left(\theta_{i}\left(1-P_{f}\right)\right) /\left(\theta_{i}\left(1-P_{f}\right)+\left(1-\theta_{i}\right) P_{m}\right)$. Generally speaking, for the greedy search algorithm, sensing errors will affect the estimation of channel free probabilities and the reward calculation, while the basic principle in the algorithm is still to select the channel with the maximal additional reward to the system. For the incremental algorithm, the average throughput calculation in each round will be affected. The detailed derivations are omitted here due to space limitations.

In this paper, the idle/busy state of a channel in a slot is independent of its states in other slots. On the other hand, when the channel idle/busy states are correlated (e.g., modeled by a two-state Markov chain), the sensing of a channel in a slot has two gains [9], [27]: a short-term gain, which is the transmission opportunity if the channel is sensed to be free, and a long-term gain, which is the updated state information of the channel (the updated state information can benefit the secondary user in later sensing selection). Our algorithm can maximize the short-term gain in a slot. However, an optimal solution needs to strike a balance between the short-term gain and the longterm gain. This means that the user may still need to sense other channels to obtain updated state information (to benefit later sensing decisions). This is an interesting research topic that deserves further significant investigation, which is also our further research direction.

\section{APPENDIX \\ Primary-Free Probability Update Procedure FOR CHANNEL $b_{l}(1 \leq l \leq k-1)$ WHEN USER 1 \\ Is SELECTING $a_{k}$}

We have four possible situations, which are listed as follows.

1) $b_{l} \notin \mathcal{A}$ : Then, $\theta_{b_{l}}^{*}=\theta_{b_{l}}$.

2) $b_{l} \in \mathcal{A}$, and $g\left(b_{l}, \mathbf{A}\right)<l$ : Since user 1 should sense channel $b_{l}$ prior to user 2 and user 1 has not stopped at channel $b_{l}$, we have $\theta_{b_{l}}^{*}=0$.

3) $b_{l} \in \mathcal{A}$, and $g\left(b_{l}, \mathbf{A}\right)=l$ (i.e., $\left.a_{l}=b_{l}\right)$ : Channel $b_{l}$ has the same sensing position (i.e., the $l$ th position) with both users.

a) If either the FAIL_THEN_QUIT or COLLIDE strategy is adopted as the contention-resolution strategy, apparently, there should be primary activity in channel $b_{l}$, because, otherwise, user 1 should have stopped at its $l$ th sensing. Therefore, $\theta_{b_{l}}^{*}=0$.

b) If FAIL_THEN_CONTINUE is adopted as the contentionresolution strategy, since user 1 has not stopped at channel $b_{l}$, either of the following two events should happen: 1) There are primary activities in channel $b_{l}$, and 2) there is no primary activity in channel $b_{l}$, users 1 and 2 both proceed to sense channel $b_{l}$ in their $l$ th sensing, and user 2 wins. Define $y$ as the scenario that event 1 happens and $x$ as the scenario that either event 1 or 2 happens. The updated primary-free probability $\theta_{b_{l}}^{*}$ is given by $\theta_{b_{l}}^{*}=1-P(y \mid x)$, which means that $P(y \mid x)=1-\theta_{b_{l}}^{*}$. Here, $P(\cdot)$ stands for the probability of an event. We also have $P(y)=1-\theta_{b_{l}}$, $P(x \mid y)=1$, and

$$
P(x)=1-\theta_{b_{l}}+\theta_{b_{l}}\left(\Pi_{j=1}^{l-1}\left(1-\theta_{b_{j}}^{*}\right)\right) \cdot 0.5
$$

where the term $\Pi_{j=1}^{l-1}\left(1-\theta_{b_{j}}^{*}\right)$ means the probability that user 2 does not stop at its first $l-1$ sensings, and the term 0.5 means that user 2 has a probability of 0.5 to win in the contention with user 1 . Based on Bayesian formula $P(y \mid x)=((P(y) P(x \mid y)) /$ $(P(x)))$, we can get

$$
\theta_{b_{l}}^{*}=\theta_{b_{l}} \frac{0.5 \Pi_{j=1}^{l-1}\left(1-\theta_{b_{j}}^{*}\right)}{1-\theta_{b_{l}}+0.5 \theta_{b_{l}} \Pi_{j=1}^{l-1}\left(1-\theta_{b_{j}}^{*}\right)} .
$$


4) $b_{l} \in \mathcal{A}$, and $g\left(b_{l}, \mathbf{A}\right)>l$ : User 2 should sense channel $b_{l}$ prior to user 1 . Since user 1 has not stopped at channel $b_{l}$, either of the following two events should happen: 1) There are primary activities in channel $b_{l}$, and 2) there is no primary activity in channel $b_{l}$, and user 2 proceeds to sense channel $b_{l}$ and transmits in channel $b_{l}$. Similarly, we can get

$$
\theta_{b_{l}}^{*}=\theta_{b_{l}} \frac{\Pi_{j=1}^{l-1}\left(1-\theta_{b_{j}}^{*}\right)}{1-\theta_{b_{l}}+\theta_{b_{l}} \Pi_{j=1}^{l-1}\left(1-\theta_{b_{j}}^{*}\right)} .
$$

\section{ACKNOWLEDGMENT}

The authors would like to thank the Associate Editor, Dr. Y.-C. Liang, and the anonymous reviewers for their constructive comments and suggestions, which helped to improve the quality of this paper.

\section{REFERENCES}

[1] S. Haykin, "Cognitive radio: Brain-empowered wireless communications," IEEE J. Sel. Areas Commun., vol. 23, no. 2, pp. 201-220, Feb. 2005.

[2] R. W. Thomas, D. H. Friend, L. A. Dasilva, and A. B. MacKenzie, "Cognitive networks: Adaptation and learning to achieve end-to-end performance objectives," IEEE Commun. Mag., vol. 44, no. 12, pp. 51-57, Dec. 2006.

[3] E. Hossain, D. Niyato, and Z. Han, Dynamic Spectrum Access in Cognitive Radio Networks. Cambridge, U.K.: Cambridge Univ. Press, 2009.

[4] J. Mitola, III and G. Q. Maguire, Jr., "Cognitive radio: Making software radios more personal," IEEE Pers. Commun., vol. 6, no. 4, pp. 13-18, Aug. 1999.

[5] Z. Han, R. Fan, and H. Jiang, "Replacement of spectrum sensing in cognitive radio," IEEE Trans. Wireless Commun., vol. 8, no. 6, pp. 28192826, Jun. 2009

[6] Y.-C. Liang, Y. Zeng, E. C. Y. Peh, and A. T. Hoang, "Sensing-throughput tradeoff for cognitive radio networks," IEEE Trans. Wireless Commun., vol. 7, no. 4, pp. 1326-1337, Apr. 2008.

[7] X. Kang, Y.-C. Liang, H. K. Garg, and L. Zhang, "Sensing-based spectrum sharing in cognitive radio networks," IEEE Trans. Veh. Technol. to be published.

[8] H. Su and X. Zhang, "Cross-layer based opportunistic MAC protocols for QoS provisionings over cognitive radio mobile wireless networks," IEEE J. Sel. Areas Commun., vol. 26, no. 1, pp. 118-129, Jan. 2008.

[9] Q. Zhao, L. Tong, A. Swami, and Y. Chen, "Decentralized cognitive MAC for opportunistic spectrum access in ad hoc networks: A POMDP framework," IEEE J. Sel. Areas Commun., vol. 25, no. 3, pp. 589-600, Apr. 2007.

[10] L. Lai, H. El Gamal, H. Jiang, and H. V. Poor, "Optimal medium access control in cognitive radios: A sequential design approach," in Proc. IEEE ICASSP, Las Vegas, NV, Mar./Apr. 2008, pp. 2073-2076.

[11] A. Motamedi and A. Bahai, "Optimal channel selection for spectrumagile low-power wireless packet switched networks in unlicensed band," EURASIP J. Wireless Commun. Netw., vol. 2008, Jan. 2008. Article ID 896420.

[12] Y. S. Chow, H. Robbins, and D. Siegmund, Great Expectations: The Theory of Optimal Stopping. Boston, MA: Houghton Mifflin, 1971.

[13] D. Zheng, W. Ge, and J. Zhang, "Distributed opportunistic scheduling for ad-hoc networks with random access: An optimal stopping approach," IEEE Trans. Inf. Theory, vol. 55, no. 1, pp. 205-222, Jan. 2009.

[14] N. B. Chang and M. Liu, "Optimal channel probing and transmission scheduling for opportunistic spectrum access," in Proc. 13th ACM Аnnu. Int. Conf. MobiCom, Montreal, QC, Canada, Sep. 2007, pp. 27-38.

[15] S. Guha, K. Munagala, and S. Sarkar, "Approximation schemes for information acquisition and exploitation in multichannel wireless networks," in Proc. 44th Annu. Allerton Conf. Commun., Control Comput., Monticello, IL, Sep. 2006.

[16] H. Jiang, L. Lai, R. Fan, and H. V. Poor, "Optimal selection of channel sensing order in cognitive radio," IEEE Trans. Wireless Commun., vol. 8, no. 1, pp. 297-307, Jan. 2009.
[17] H. Kim and K. G. Shin, "Fast discovery of spectrum opportunities in cognitive radio networks," in Proc. IEEE Symp. New Frontiers DySPAN, Chicago, IL, Oct. 2008.

[18] J. Jia, Q. Zhang, and X. Shen, "HC-MAC: A hardware-constrained cognitive MAC for efficient spectrum management," IEEE J. Sel. Areas Commun., vol. 26, no. 1, pp. 106-117, Jan. 2008.

[19] C. Cordeiro, K. Challapali, and M. Ghosh, "Cognitive PHY and MAC layers for dynamic spectrum access and sharing of TV bands," in Proc. 1st Int. Workshop TAPAS, Boston, MA, Aug. 2006.

[20] L. X. Cai, X. Shen, J. W. Mark, L. Cai, and Y. Xiao, "Voice capacity analysis of WLAN with unbalanced traffic," IEEE Trans. Veh. Technol., vol. 55, no. 3, pp. 752-761, May 2006.

[21] P. Wang, H. Jiang, and W. Zhuang, "A new MAC scheme supporting voice/data traffic in wireless ad hoc networks," IEEE Trans. Mobile Comput., vol. 7, no. 12, pp. 1491-1503, Dec. 2008.

[22] H. Jiang, P. Wang, W. Zhuang, and H. V. Poor, "Service time analysis of a distributed medium access control scheme," IEEE Trans. Wireless Commun., vol. 7, no. 10, pp. 3988-3998, Oct. 2008.

[23] H. Luo, J. Cheng, and S. Lu, "Self-coordinating localized fair queueing in wireless ad hoc networks," IEEE Trans. Mobile Comput., vol. 3, no. 1, pp. 86-98, Jan.-Mar. 2004.

[24] H. Jiang, W. Zhuang, X. Shen, and Q. Bi, "Quality-of-service provisioning and efficient resource utilization in CDMA cellular communications," IEEE J. Sel. Areas Commun., vol. 24, no. 1, pp. 4-15, Jan. 2006.

[25] V. Kanodia, A. Sabharwal, and E. Knightly, "MOAR: A multi-channel opportunistic auto-rate media access protocol for ad hoc networks," in Proc. 1st Annu. Int. Conf. BROADNETS, San Jose, CA, Oct. 2004, pp. 600-610.

[26] A. Sabharwal, A. Khoshnevis, and E. Knightly, "Opportunistic spectral usage: Bounds and a multi-band CSMA/CA protocol," IEEE/ACM Trans. Netw., vol. 15, no. 3, pp. 533-545, Jun. 2007.

[27] L. Lai, H. El Gamal, H. Jiang, and H. V. Poor, "Optimal medium access protocols for cognitive radio networks," in Proc. 6th Int. Symp. Model. Optim. Mobile, Ad Hoc, Wireless Netw. (WiOpt'08), Mar./Apr. 2008, pp. 328-334.

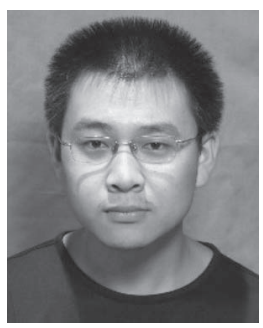

Rongfei Fan received the B.E. degree in electrical engineering from Harbin Institute of Technology, Harbin, China, in 2007. He is currently working toward the Ph.D. degree with the Department of Electrical and Computer Engineering, University of Alberta, Edmonton, AB, Canada.

His research interests include cognitive radio networking and cross-layer design.

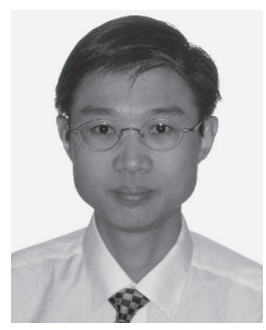

Hai Jiang (S'04-M'07) received the B.S. and M.S. degrees in electronics engineering from Peking University, Beijing, China, in 1995 and 1998, respectively, and the Ph.D. degree (with an Outstanding Achievement in Graduate Studies Award) in electrical engineering from the University of Waterloo, Waterloo, ON, Canada, in 2006.

Since July 2007, he has been an Assistant Professor with the Department of Electrical and Computer Engineering, University of Alberta, Edmonton, AB, Canada. His research interests include radio resource management, cognitive radio networking, and cross-layer design for wireless multimedia communications.

Dr. Jiang is an Associate Editor for the IEEE TRANSACTIONS ON Vehicular TeChnology. He served or will serve as a Cochair for the General Symposium at the International Wireless Communications and Mobile Computing Conference in 2007, the Communications and Networking Symposium at the Canadian Conference on Electrical and Computer Engineering in 2009, and the Wireless and Mobile Networking Symposium at the IEEE International Conference on Communications in 2010. He received an Alberta Ingenuity New Faculty Award in 2008 and a Best Paper Award from the IEEE Global Communications Conference in 2008. 